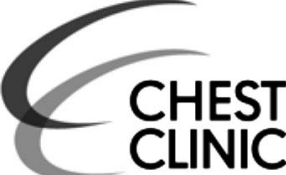

\title{
Journal club summary page
}

\author{
Benjamin Prudon
}

\section{PATIENT FACTORS ASSOCIATED WITH 'LOSS TO FOLLOW-UP' AFTER STARTING TB THERAPY WITHIN THE UK}

Completion of a full treatment programme for the management of tuberculosis is pivotal in achieving successful disease treatment and preventing development of drug resistant disease. In this study (Epidemiol Infect 2013;141:1223-1231), investigators compared characteristics of patients in the UK between 2001 and 2007 who completed TB therapy to those who were lost to follow-up. Factors associated with an increased risk of loss to follow-up included being male; adjusted odds ratio (aOR 1.29); having sputum smear-positive disease (aOR 1.25), and arriving in the UK within two years (aOR 3.58) particularly those of White ethnic origin (aOR 6.39).

\section{THE ADDITION OF C REACTIVE PROTEIN (CRP) CONCENTRATIONS TO CLINICAL FEATURES IMPROVES PREDICTIVE TOOLS FOR DIAGNOSING PNEUMONIA IN PRIMARY CARE}

This Europe-wide study (BMJ 2013;346: f2450 doi:10.1136/bmj.f2450) of patients presenting to primary care with acute cough investigated the additional benefit of adding either CRP or procalcitonin concentrations to clinical features for predicting the diagnosis of pneumonia. Data from 2820 patients were analysed, and $140(5 \%)$ were identified as having pneumonia. The optimal combination of clinical prediction items included absence of rhinorrhoea and presence of breathlessness, crackles and diminished breath sounds on auscultation, with tachycardia, and fever; (ROC area of 0.70). Addition of CRP at the optimal cut off of $>30 \mathrm{mg} /$ $\mathrm{L}$ increased the ROC area to 0.77 . Measurement of procalcitonin did not improve diagnostic accuracy.

\section{PALIVIZUMAB TREATMENT IMPACT ON INFANT WHEEZE}

Respiratory syncytial virus (RSV) infection is associated with subsequent recurrent wheeze in infants, but it remains unclear if RSV is the direct cause of this. Severe RSV infection risk

Correspondence to Benjamin Prudon, Clinical Research Associate, Newcastle Regional Sleep Service, Freeman Hospital, Freeman Road, Newcastle upon Tyne, NE7 7DN, UK; ben_prudon@doctors.org.uk can be reduced through treatment with the monoclonal antibody palivizumab. This placebo-controlled trial (N Engl J Med 2013;368:1791-1799) compared preterm infants born at a gestational age of 33 to 35 weeks given monthly palivizumab injections (214 infants) or placebo (215 infants) during the RSV season. During the follow-up period of 12 months, the proportion of infants with recurrent wheeze was significantly lower in treatment group compared to control, $(11 \%$ vs $21 \%, \mathrm{p}=0.01)$.

\section{INTERSTITIAL LUNG DISEASE CHARACTERISTICS OF ANTI-JO-1 ASSOCIATED ANTISYNTHETASE SYNDROME}

Antisynthetase syndrome is an inflammatory myopathy associated with the presence of antisynthetase antibodies such as anti-Jo-1. Interstitial lung disease (ILD) is a frequent cause of morbidity and mortality within this disease. In this study (Arthritis Care \& Res 65;2013:800-808), characteristics of all patients diagnosed with anti-Jo-1 disease at four French centres between 1998-2010 were evaluated. Ninety-one patients were identified of which 66 (73\%) had ILD; COP (n 11), NSIP $(n=39)$, and UIP $(n=16)$. Clinical manifestations of the pulmonary disease included acute onset ILD $(n=12)$, symptomatic progressive onset $(n=35)$, and asymptomatic $(n=19)$. Following antiinflammatory therapy, ILD resolved completely in 16 patients, improved and stabilised in 28 patients, with progressive deterioration in 11 patients. Factors associated with ILD deterioration included a HRCT pattern of UIP, age $\geq 55$ years, and respiratory muscle involvement.

\section{TB TREATMENT PROGRAMMES CAN WITHSTAND HURRICANES}

The Centres for Disease Control and Prevention have reported (JAMA 2013;309:1766-1767) that despite Hurricane Sandy causing significant environmental and infrastructure damage to areas in North-East USA during October 2012, TB programme officials were able to account for all the patients and there was no significant disruption to treatment plans. This success with attributed to learning from experiences following
Hurricane Katrina; lists of patients who may be affected by the storm were prepared, patients on directly observed therapy (DOT) were provided with a supply of medication in case DOT was disrupted, and contact numbers for the Health Department were provided to patients to allow notification of changes to contact information.

\section{PULMONARY EMBOLUS MORTALITY}

Malignancy is known to be a significant risk factor for mortality from acute pulmonary embolism. A large retrospective audit of 1596 patients ( $\mathrm{J}$ Thorac Imaging 2013;28:196201) has further clarified predictors of 30 -day mortality in those with and without malignancy. Analysis showed patients with known malignancy had a high 30-day mortality rate (19.6\% vs 3.2\%, p < 0.001). However, there were fewer predictors of morality for those with malignancy than without. Advanced age, presence of coronary artery disease, history of stroke, and COPD were all significantly more predictive of death in those without malignancy but not for those with. The presence of an enlarged right ventricle on CTPA (right to left ventricular diameter ratio $>1.0$ ) was associated with a higher risk of 30-day death only among subjects with no known malignancy at the time of the CTPA $(\mathrm{OR}=4.08,95 \%$ CI 1.7 to 10.0$)$.

\section{NUTRITIONAL STRATEGIES FOR MECHANICALLY VENTILATED}

\section{PATIENTS WITH ACUTE LUNG INJURY}

Optimal nutritional strategies for mechanically ventilated patients with Acute Lung Injury remains uncertain with two opposing strategies having supporting literature; full energy enteral feeding and low energy permissive underfeeding. The EDEN randomised-controlled-trial (JAMA 2012;307:795-803) previously compared the two strategies over six weeks and showed no difference in short term outcomes. To investigate the possibility that a superior nutritional strategy may produce improvements in long term outcomes, patients participating within the EDEN trial completed further assessments at six and 12 months after randomisation (BMJ 2013;346: f1532 doi:10.1136/bmj.f1532). At these assessment points there were no significant differences in physical, psychological or quality of lifemeasurements between patientgroups.

\section{Competing interests None.}

Provenance and peer review Commissioned; internally peer reviewed.

To cite Prudon B. Thorax 2013;68:700.

Thorax 2013;68:700.

doi:10.1136/thoraxjnl-2013-203936 